\title{
REDUCING THE RANK OF $(A-\lambda B)^{1}$
}

\author{
GERALD L. THOMPSON AND ROMAN L. WEIL
}

Abstract. The rank of the $n \times n$ matrix $(A-\lambda I)$ is $n-J(\lambda)$ when $\lambda$ is an eigenvalue occurring in $J(\lambda) \geqq 0$ Jordan blocks of the Jordan normal form of $A$. In our principal theorem we derive an analogous expression for the rank of $(A-\lambda B)$ for general, $m \times n$, matrices. When $J(\lambda)>0, \lambda$ is a rank-reducing number of $(A-\lambda I)$. We show how the rank-reducing properties of eigenvalues can be extended to $m \times n$ matrix expressions $(A-\lambda B)$. In particular we give a constructive way of deriving a polynomial $P(\lambda, A, B)$ whose roots are the only rank-reducing numbers of $(A-\lambda B)$. We name this polynomial the characteristic polynomial of $A$ relative to $B$ and justify that name.

The rank of the $n \times n$ matrix $(A-\lambda I)$ is $n-J(\lambda)$ when $\lambda$ is an eigenvalue appearing on the main diagonal of $J(\lambda) \geqq 0$ distinct Jordan blocks in the Jordan normal form of $A$. See, e.g., Wilkinson [2, p. 11]. Thus an eigenvalue, $\lambda$, of $A$ is a rank-reducing number of $(A-\lambda I)$.

In this paper we show how the rank-reducing properties of eigenvalues can be extended to $m \times n$ matrix expressions $(A-\lambda B)$. In particular we give a constructive way of deriving a polynomial $P(\lambda, A, B)$ whose roots are the only rank-reducing numbers of $(A-\lambda B)$. We have named this polynomial the characteristic polynomial of $A$ relative to $B$. In our principal theorem we derive a simple expression for the rank of $(A-\lambda B)$. Finally we relate our results to the normal form derived by Gantmacher.

Consider the rank-reducing scalars $\lambda$ of $(A-\lambda B)$. If the determinant $|B| \neq 0$, then the problem of finding rank-reducing numbers is equivalent to an ordinary eigenvalue problem. For if $|B| \neq 0$, the rank of $(A-\lambda B)$ is the same as that of $\left(B^{-1} A-\lambda I\right)$ and the rank-reducing $\lambda$ of $(A-\lambda B)$ are the eigenvalues of $B^{-1} A$. But this analysis breaks down if $B$ is singular or, more generally, if $A$ and $B$ are rectangular. In this paper we shall consider rectangular $A$ and $B$, $m \times n$. Let $\operatorname{rank}(A)=p$ and $\operatorname{rank}(B)=r$.

Lemma 1. For all $m \times n A$ and $B$, there exist nonsingular $S_{1}$ and $T_{1}$

Received by the editors January $26,1970$.

AMS 1969 subject classifications. Primary 1505, 1525, 6540.

Key words and phrases. Eigenvalues, characteristic polynomial, rank.

1 Research support was provided by the U.S. Office of Naval Research under Contract NONR 760(24) NR 047-048 to Carnegie-Mellon University and by the National Science Foundation via a grant to the University of Chicago. 
such that $S_{1}(A-\lambda B) T_{1}$ can be partitioned into

$$
\begin{array}{r}
r \\
s\left[\begin{array}{llll}
E_{11} & E_{12} & 0 & 0 \\
E_{21} & 0 & 0 & 0 \\
0 & 0 & I_{q} & 0 \\
0 & 0 & 0 & 0
\end{array}\right]-\lambda\left[\begin{array}{llll}
I_{r} & 0 & 0 & 0 \\
0 & 0 & 0 & 0 \\
0 & 0 & 0 & 0 \\
0 & 0 & 0 & 0
\end{array}\right]
\end{array}
$$

where $q \leqq \min (m-r, n-r, p), E_{12}$ is in column echelon form and rank $\left(E_{12}\right)=t$, and $E_{21}$ is in row echelon form and rank $\left(E_{21}\right)=s$. Any of the numbers $s, t, q$, may be zero and $r+s+q \leqq m, r+t+q \leqq n$.

Proof. In Theorem 2.2 of [1] we proved a similar statement. An outline of the proof is as follows. In the expression $A-\lambda B$ use elementary row and column operations to put $B$ in diagonal form, ignoring their effects on $A$. Thus $A-\lambda B$ becomes

$$
\left[\begin{array}{ll}
C_{11} & C_{12} \\
C_{21} & C_{22}
\end{array}\right]-\lambda\left[\begin{array}{ll}
I_{r} & 0 \\
0 & 0
\end{array}\right] .
$$

Now do the same kind of operation on $C_{22}$, except putting the identity matrix last so that $C_{22}$ becomes

$$
s\left[\begin{array}{cc}
t & q \\
q & 0 \\
0 & I_{q}
\end{array}\right] .
$$

By further row and column operations (in particular transferring all zero rows to the bottom and all zero columns to the right) change $C_{12}$ to $\left(E_{12} 0\right)$ where $E_{12}$ is in column echelon form and change $C_{21}$ to $\left[\begin{array}{c}E_{21} \\ 0\end{array}\right]$ where $E_{21}$ is in row echelon form. Note that each successive level of transformation does not destroy the results of the preceding transformations.

LEMMA 2. If $s+t>0$ in (1), then there are nonsingular $S_{2}$ and $T_{2}$ such that $S_{2}(A-\lambda B) T_{2}$ can be partitioned into

$$
r+t\left[\begin{array}{lllll}
C_{11} & 0 & 0 & 0 & 0 \\
0 & 0 & I_{t} & 0 & 0 \\
0 & I_{s} & 0 & 0 & 0 \\
0 & 0 & 0 & I_{q} & 0 \\
0 & 0 & 0 & 0 & 0
\end{array}\right]-\lambda\left[\begin{array}{lllll}
D_{11} & D_{12} & 0 & 0 & 0 \\
D_{21} & D_{22} & 0 & 0 & 0 \\
0 & 0 & 0 & 0 & 0 \\
0 & 0 & 0 & 0 & 0 \\
0 & 0 & 0 & 0 & 0
\end{array}\right] .
$$


Proof. In Theorem 2.4 of [1] we prove a similar statement. An outline of the proof is as follows. Permute and partition $E_{12}$ and $E_{21}$ in (1) so that the first $2 \times 2$ block of (1) becomes

$$
{ }_{s}^{r-t}\left[\begin{array}{lll}
F_{11} & F_{12} & F_{13} \\
F_{21} & F_{22} & I_{t} \\
F_{31} & I_{s} & 0
\end{array}\right]-\lambda\left[\begin{array}{lll}
P_{11} & P_{12} & 0 \\
P_{21} & P_{22} & 0 \\
0 & 0 & 0
\end{array}\right]
$$

where $P$ is a permutation matrix and $I_{t}$ and $I_{t}$ are constructed from the leading ones in the echelon forms $E_{12}$ and $E_{21}$. Further row and column operations lead to (2) where the $(r-t) \times(r-s)$ matrices $C_{11}$ and $D_{11}$ are defined by

$$
\begin{aligned}
& C_{11}=F_{11}-F_{12} F_{31}-F_{13} F_{21}+F_{13} F_{22} F_{31}, \\
& D_{11}=P_{11}-P_{12} F_{31}-F_{13} P_{21}+F_{13} P_{22} F_{31} .
\end{aligned}
$$

Lemma 3. (a) If $s+t=0$ in (1), then any number $\lambda$ reduces the ranks of both $(A-\lambda B)$ and $\left(E_{11}=\lambda I\right)$ by the number of Jordan blocks containing that $\lambda$ in the Jordan normal form of $E_{11}$.

(b) If $s+t>0$, then $\lambda$ is a rank-reducing number for $(A-\lambda B)$ if and only if it is a rank-reducing number for $\left(C_{11}-\lambda D_{11}\right)$ in (2).

PROOF. (a) If $s+t=0$, then the second row and second column of (1) are empty and the result is an obvious consequence of the classical results on the Jordan normal form; e.g., see [2].

(b) Suppose $s+t>0$. Define the row vector

$$
w=\left(w_{1}, w_{2}, w_{3}, w_{4}, w_{5}\right)
$$

partitioned the same way the rows of (2) are. Multiplying $S_{2}(A-\lambda B) T_{2}=0$ by $w$, we obtain from expression (2) the equations

$$
\begin{array}{rlrl}
w_{1}\left(C_{11}-\lambda D_{11}\right)-w_{2} \lambda D_{21} & =0, \\
-w_{1} \lambda D_{12} & -w_{2} \lambda D_{22}+w_{3} I_{s} & & =0, \\
w_{2} I_{t} & & =0, \\
& & w_{4} I_{q} & =0 .
\end{array}
$$

Here $w_{5}$ is arbitrary and can be ignored. From (6), $w_{4}=0$ and from (5), $w_{2}=0$. The solution of (4) determines $w_{3}\left(=w_{1} \lambda D_{12}\right)$ and imposes no constraint on $w_{1}$. Hence equations (3) to (6) reduce to simply $w_{1}\left(C_{11}-\lambda D_{11}\right)=0$, regardless of the value of $\lambda$. From this it follows that the row rank (and hence the rank) of $(A-\lambda B)$ and $\left(C_{11}-\lambda D_{11}\right)$ satisfy 


$$
\operatorname{rank}(A-\lambda B)=\operatorname{rank}\left(C_{11}-\lambda D_{11}\right)+q+s+t
$$

from which the lemma follows.

Lemma 4. Assume $(A-\lambda B)$ has at least one rank-reducing number. Then either $s+t=0$ in (1) or there is a strictly smaller square matrix $\left(A^{(k)}-\lambda B^{(k)}\right)$ such that $s_{k}+t_{k}=0, B^{(k)}$ is an identity matrix, and $\lambda$ is a rank-reducing number for $(A-\lambda B)$ if and only if it is a rank-reducing number for $\left(A^{(k)}-\lambda B^{(k)}\right)$.

Proof. If $s+t>0$, define $\left(A^{(1)}-\lambda B^{(1)}\right) \equiv(A-\lambda B)$ and define $\left(A^{(2)}-\lambda B^{(2)}\right) \equiv\left(C_{11}-\lambda D_{11}\right)$, where $C_{11}$ and $D_{11}$ are defined in (2) of Lemma 2. Note that $\left(A^{(2)}-\lambda B^{(2)}\right)$ is of dimension strictly smaller than that of $\left(A^{(1)}-\lambda B^{(1)}\right)$; by Lemma 3 these two matrices have the same rank-reducing numbers. Now put $\left(A^{(2)}-\lambda B^{(2)}\right)$ in to its normal form (1) and let its block dimensions be $r_{2}, s_{2}, t_{2}$, and $q_{2}$. If $s_{2}+t_{2}=0$, the lemma is proved; and if $s_{2}+t_{2}>0$ we can reapply the same procedure and define a strictly smaller matrix $\left(A^{(8)}-\lambda B^{(3)}\right)$ that has the same rank-reducing numbers, and so on. At the $j$ th step construct $\left(A^{(j)}-\lambda B^{(j)}\right)$ whose size is $\left(r_{j}+s_{j}\right) \times\left(r_{j}+t_{j}\right)$ where $r_{j}+s_{j}=r_{j-1}-t_{j-1}$ and $r_{j}+t_{j}=r_{j-1}-s_{j-1}$. Since dimensions are reduced at each step the construction process must obviously stop after a finite number of steps with a matrix $\left(A^{(k)}-\lambda B^{(k)}\right)$ such that $s_{k}+t_{k}=0$. Because of the assumption that there is a rank-reducing number, it follows that $r_{k}>0$ and $B^{(k)}$ is an identity matrix. By the construction and Lemma 3 , the rank-reducing numbers of $(A-\lambda B)$ are the same as those of $\left(A^{(k)}-\lambda B^{(k)}\right)$.

REMARK 1. If $(A-\lambda B)$ has no rank-reducing numbers, the procedure outlined in the proof of Lemma 4 can be carried out, but will terminate at the $(k+1)$ st stage whenever $r_{k}=s_{k}$ or $r_{k}=t_{k}$ or both.

REMARK 2. When $s_{k}+t_{k}=0,\left(A^{(k)}-\lambda B^{(k)}\right)$ is square and $B^{(k)}$ is an identity matrix. Hence if $(A-\lambda B)$ has at least one rank-reducing number, the procedure outlined in the proof of Lemma 4 will lead to an ordinary eigenvalue problem $\left(A^{(k)}-\lambda B^{(k)}\right)$ where $B^{(k)}$ is an identity matrix. Therefore all rank-reducing numbers of $\left(A^{(k)}-\lambda B^{(k)}\right)$, and those of $(A-\lambda B)$ can be found by using ordinary eigenvalue techniques.

Definition 1. For $m \times n$ matrices $A$ and $B$ we define the characteristic polynomial $P(\lambda, A, B)$ of $A$ relative to $B$ as follows:

(a) If there are no rank-reducing numbers of $(A-\lambda B)$, then $P(\lambda, A, B)=1$.

(b) If there is at least one rank-reducing number of $(A-\lambda B)$, let $P(\lambda, A, B)$ be the characteristic polynomial of $E_{11}^{(\mathbf{k})}$ which is given 
by the normal form of $A^{(k)}-\lambda B^{(k)}$ where $k$ is the smallest such that $s_{k}+t_{k}=0$. (Note that $E_{11}^{(1)}$ is the same as $E_{11}$ in (1).)

Justification for the name characteristic polynomial is given below.

Definition 2. For any complex number $\lambda$ define

(a) $J(\lambda)=0$ if $\lambda$ is not a root of $P(\lambda, A, B)=0$,

(b) $J(\lambda)=$ the number of Jordan blocks containing $\lambda$ in the Jordan normal form of $E_{11}^{(k)}$ where $k$ is the smallest such that $s_{k}+t_{k}=0$.

THEOREM. For any complex number $\lambda$

$$
\operatorname{rank}(A-\lambda B)=r+q-J(\lambda)
$$

where $r$ and $q$ are defined in (1).

PRoof. If $\lambda$ is not a rank-reducing number, the rank of the submatrix

$$
\left[\begin{array}{cc}
E_{11}-\lambda I_{r} & E_{12} \\
E_{21} & 0
\end{array}\right]
$$

in (1) is $r$, so $\operatorname{rank}(A-\lambda B)=r+q$. If $\lambda$ is a rank-reducing number then repeated applications of Lemmas 3 and 4 show that $\lambda$ reduces the rank of the derived $\left(E_{11}^{(k)}-\lambda I_{r_{k}}\right)$ matrix by $J(\lambda)$ and hence reduces the rank of $(A-\lambda B)$ by $J(\lambda)$. This completes the proof.

Obviously the Cayley-Hamilton theorem, which states that a square matrix satisfies its own characteristic polynomial equation, cannot be extended to rectangular matrices since powers $(>1)$ of a rectangular matrix are not defined. However, the less emphasized property of a square matrix $A$ that the roots of $P(\lambda, A, I)=0$ are identical with the rank-reducing numbers of $(A-\lambda I)$ can be extended to rectangular matrices as we have shown in this paper. Thus the corresponding fact that the roots of $P(\lambda, A, B)=0$ are identical with the rank-reducing numbers of $(A-\lambda B)$ provides the justification for calling $P(\lambda, A, B)$ the characteristic polynomial of $A$ with respect to $B$.

The matrix $(A-\lambda B)$ has been called a matrix pencil. Gantmacher [1, Chapter 12] develops a theory of matrix pencils and applies his results to quadratic forms and differential equations. Gantmacher transforms the $m \times n$ matrix $(A-\lambda B)$ to a rational, Jordan-like normal form as follows. Let the nonsingular $m \times m$ matrix $S$ and the nonsingular $n \times n$ matrix $T$ represent a series of elementary row and column operations, respectively. Then there exist $S$ and $T$ so that $S(A-\lambda B) T$ is (rectangular) block diagonal with as many as $(p+q+r+2)$ blocks: 


$$
\left\{h[\stackrel{g}{0}], L_{\epsilon_{0+1}}, \cdots, L_{\epsilon_{0+p}}, L_{\eta h+1}^{T}, \cdots, L_{\eta_{h+q}}^{T}, N_{\mu_{1}}, \cdots, N_{\mu_{r}},(J-\alpha I)\right\} .
$$

All other entries in $S(A-\lambda B) T$ are zero. Neither the first nor the last block need exist for a given system nor need $p, q, r$ be positive. The matrix $S(B-\alpha A) T$ is the "direct sum" of the diagonal blocks. The block

$$
h[\stackrel{g}{0}]
$$

has $h$ rows and $g$ columns and all its elements are zero. The block $L_{\epsilon_{g+i}}$ has $\epsilon_{g+i}$ rows and $\epsilon_{o+i}+1$ columns with structure

$$
L_{\epsilon}=\left[\begin{array}{cccccc}
\lambda & 1 & & & & 0 \\
& \lambda & & 1 & & \\
& & \cdot & & \cdot & \\
0 & & \cdot & & \cdot & \\
& & & \lambda & & \\
& & & & & \\
& & &
\end{array}\right]
$$

and rank $\epsilon$. The block $L_{\eta_{h+j}}^{T}$ has $\left(\eta_{h+j}+1\right)$ rows and $\eta_{h+j}$ columns with structure

$$
L_{\eta}^{T}=\left[\begin{array}{lllllll}
\lambda & & & & & 0 \\
1 & \lambda & & & & \\
& 1 & & & & \\
& & & . & & & \\
& & & \cdot & & \\
& & & 1 & & \\
& & & &
\end{array}\right]
$$

and rank $\eta$. The square block $N_{\mu_{k}}$ is $\mu_{k}$ by $\mu_{k}$ with structure

$$
N_{\mu}=\left[\begin{array}{lllll}
1 & \mu & & \\
& \lambda & & 0 \\
& 1 & \cdot & \\
& & \ddots & \lambda \\
& & & \lambda \\
& & & 1
\end{array}\right]
$$

and rank $\mu$. The final block $J-\alpha I$ is an ordinary square eigensystem in Jordan normal form.

The Jordan normal form of the matrix $\left(A^{(k)}-\lambda B^{(k)}\right)$ derived by our techniques when $s_{k}+t_{k}=0$ is identical to the matrix $(J-\lambda I)$ in the Gantmacher normal form. The ranks of the matrices $L, L^{T}$, and 
$N$ of the Gantmacher normal form are independent of $\lambda$, so these matrices do not have rank-reducing numbers. Therefore, the only rank-reducing numbers of $(A-\lambda B)$ are those of $(J-\lambda I)$. Of course the rank-reducing numbers of $(J-\lambda I)$ are the eigenvalues of $J$.

\section{REFERENCES}

1. F. R. Gantmacher, The theory of matrices. Vol. 2, GITTL, Moscow, 1953; English transl., Chelsea, New York, 1959. MR 16, 438; MR 21 \#6372c.

2. Gerald L. Thompson and Roman L. Weil, The roots of matrix pencils $(A y=\lambda B y):$ Existence, calculations, and relations to game theory, Management Sciences Research Report No. 172, Graduate School of Industrial Administration, CarnegieMellon University, Report \#6936 of the Center for Mathematical Studies in Business and Economics of the University of Chicago, Chicago, Ill., 1969; Linear Algebra and Appl. (submitted).

3. J. H. Wilkinson, The algebraic eigenvalue problem, Clarendon Press, Oxford, 1965. MR 32 \#1894.

Carnegie-Mellon University, Pittsburgh, Pennsylvania 15213

University of Chicago, Chicago, Illinois 60637 